\title{
PESO DE HOJAS COMO HERRAMIENTA PARA ESTIMAR EL ÁREA FOLIAR EN SOYA*
}

\author{
${ }^{\bullet}$ Felipe Garcés Fiallos ${ }^{1,2}$ y Carlos Alberto Forcelini ${ }^{3}$ \\ * Parte de la tesis de Maestría en Agronomía con Área en Fitopatología del primer autor. \\ ${ }^{1}$ Becario de la Secretaría Nacional de Educación Superior, Ciencia, Tecnología e Innovación del Ecuador - SENESCYT \\ ${ }^{2}$ Unidad de Investigación Cientifica y Tecnológica, Universidad Técnica Estatal de Quevedo, km 7 vía \\ Quevedo - El Empalme, C. P. 73. Mocache,Los Ríos, Ecuador. ${ }^{\circ}$ felipegarces23@yahoo.com \\ ${ }^{3}$ Universidad de Passo Fundo. Laboratorio de Fitopatología, CEP 99052-900, Passo Fundo, RS, Brasil
}

Resumen

$\mathrm{C}$ uantificaciones del área foliar en plantas son importantes en estudios de daños ocasionados por enfermedades, por lo tanto su determinación requiere el uso de equipamientos que no siempre se encuentran disponibles para todos. La utilización de determinaciones indirectas, como el peso de materia fresca o seca podría ayudar en este proceso. En este trabajo, se evaluó la relación entre el peso de hojas y área foliar, a partir de plantas recolectadas en el estadío R7.1, en 64 parcelas de campo con el cultivar de soja Nidera 5909 RG. El peso fresco fue medido luego de la colecta, el peso seco después de 48 horas de incubación a $65^{\circ} \mathrm{C}$ y el área foliar a través de un integralizador digital Licor. Fueron obtenidas ecuaciones significativas $\left(\mathrm{p}<0.0001\right.$ e $\mathrm{R}^{2} \mathrm{de}$ 0.74 a 0.97 ) para cada estrato y para la planta entera. Para la media de la planta, la relación de área foliar fue de $y=45.53 x+19.03$ para peso fresco e $y=176.17$ $\mathrm{x}-75.30$ para peso seco. Esta herramienta se presenta potencialmente viable para estimar el área foliar de la planta. La utilización del peso seco es mas trabajosa, más no requiere pesaje de las hojas inmediatamente después de su colecta. La utilización futura de esta herramienta requiere estudios adicionales con otros cultivares a fin de verificarse si el comportamiento es similar.

Palabras claves: Glycine max L., estratos, superficie de hoja verde

Abstract

Weight of leaves as a tool for estimating the soybean leaf area

$\mathrm{M}$ easurements of leaf area are important in yields damage studies, but the equipment needed may not be available for every one. Therefore, the use indirect comparisons with weight of fresh or dried leaves may help in estimating leaf area. This research studied the relationship between leaf weight and leaf area in plants sampled at the growth stage R7.1 from 64 field plots of Nidera 5909 RG soybean cultivar. The leaves were weighted soon after sampling for fresh weight and again after 48 hours of incubation at $65^{\circ} \mathrm{C}$ for dried weight. Leaf area was measured with a Licor digital leaf area meter. The model equations were significant ( $\mathrm{p}<0.0001, \mathrm{R}^{2} 0.74$ to 0.97 ) for each plant part (lower, medium, or upper third) and for the whole plant. In average, leaf area (y) was estimated as $\mathrm{y}=45.53 \mathrm{x}+$ 19.03 from fresh weight $(\mathrm{x})$ and as $\mathrm{y}=176.17 \mathrm{x}-75.30$ for dried weight $(\mathrm{x})$. This tool was shown potentially viable to estimate plant leaf area. The method based on dried weight is more labor consuming but does not require leaves being weight soon after sampling. The use of such comparisons for other soybean cultivars may require additional validation studies.

Key words: Glycine max L., canopies, green leaf area.

\section{INTRODUCCIÓN}

E 1 cultivo de soya tiene importancia mundial, por ser una oleaginosa que sirve para la alimentación humana y animal. En Brasil y en todos los lugares donde este cultivo es plantado, existe importante desfolia (pérdida de hojas), sea esta por factores bióticos (enfermedades y plagas) o abióticos (agua,

Recibido: 21-Noviembre-2010. Recibido en forma corregida: 03Febrero-2011. Aceptado: 22-Junio-2011.

Publicado como ARTíCULO en Ciencia y Tecnología 4(1): 13-18. 2011 luz, etc.), lo cual fue objeto de estudios como los de Jesus Junior et al. (2001), Barros et al. (2002), Pelluzio et al. (2002), Heiffig et al. (2006) y Rezende (2007).

Algunas variables como daños en la producción, pérdidas financieras, reducción del área foliar en cualquier clase de cultivos, es muy importante conocer o saber, así también evaluaciones de área foliar agregan información importante al análisis de daños por enfermedades. Según Waggoner y Berger (1987), los índices de área foliar tienen mejor correlación 
con el rendimiento de la planta, que la severidad de la enfermedad. Mediciones del área foliar a lo largo del ciclo del cultivo, o en períodos específicos, también permiten determinar la duración del área foliar y así comprender mejor los efectos de las enfermedades sobre el rendimiento de la planta.

El crecimiento de la planta de soya puede ser cuantificado por dos factores: peso seco y área foliar total de la planta (Seem, 1988). En la cuantificación de producción, la variable más relevante de crecimiento de una planta es el área foliar por unidad de área de terreno, conocido como índice de área foliar (IAF) (Bergamin Filho y Amorim, 1996).

También para evaluar los efectos derivados del control químico, se relaciona la producción con el área foliar enferma (severidad) o asimismo el área foliar removida (desfolia) (Vanderplank, 1963). El cálculo del IAF de un cultivo requiere información sobre la densidad de plantas por metro cuadrado y sobre el área foliar media de cada planta. La densidad es fácilmente obtenida a partir del conteo del número de plantas en puntos diferentes de la plantación. La obtención del área foliar media de la planta es un proceso más trabajoso. El método más preciso es por medio de aparatos integralizadores de área foliar, los cuales posee un sensor conectado a una correa por donde las hojas son conducidas y medidas. Hay también formas indirectas que permiten estimar el área foliar de un cultivo a través de su reflectancia, midiendo la intensidad de la radiación incidente y reflejada, sobre la copa, de ocho longitudes de onda utilizando un radiómetro de múltiplo espectro (Aquino et al., 1992), o también midiendo la interceptación de luz en la copa de un cultivo o que atraviesa el dosel del mismo, utilizando para esto polímetros (Díaz et al., 2005).

La necesidad de estos equipamientos limita la utilización del área foliar como variable en estudios de daños por enfermedades, a pesar de su importancia. Por eso, métodos más simples podrían ser empleados, desde que sean obtenidos a través de un integralizador de área foliar. El peso fresco o seco de las hojas potencialmente puede ser útil para tales determinaciones.

El objetivo de este trabajo fue evaluar la relación de peso fresco y seco con el área foliar de plantas de soya, verificando también si esta técnica simple y de bajo costo puede ser utilizada para estimar el índice del área foliar.

\section{Materiales y Métodos}

Dara la conducción de este trabajo, se utilizaron folíolos de soya del cultivar Nidera 5909 RG,

Cuadro 1. Estadios fonológicos y fechas de aplicación de fungicidas. Facultad de Agronomía y Medicina Veterinaria/UPF, Época de verano 2009/10

\begin{tabular}{clll}
\hline Tratamientos & \multicolumn{1}{c}{ Fungicida } & Estadío* $^{*}$ & Fecha \\
\hline 1 & $\begin{array}{l}\text { Sin aplicación de } \\
\text { fungicida en parte aérea }\end{array}$ & & \\
\hline 2 & & $\mathrm{~V} 9$ & $22 /$ enero \\
3 & & $\mathrm{~V} 9+\mathrm{R} 4$ & $22 /$ enero, $11 /$ febrero \\
4 & & $\mathrm{~V} 9+\mathrm{R} 4+\mathrm{R} 5.3$ & $22 /$ enero, $11 /$ febrero, 3/marzo \\
5 & Tebuconazol & $\mathrm{V} 9+\mathrm{R} 5.3$ & $22 /$ enero, 3/marzo \\
6 & & $\mathrm{R} 4+\mathrm{R} 5.3$ & $11 /$ febrero, 3/marzo \\
7 & & $\mathrm{R} 4$ & $11 /$ febrero \\
8 & & $\mathrm{R} 5.3$ & $3 /$ marzo \\
\hline 9 & & $\mathrm{~V} 9$ & $22 /$ enero \\
10 & & $\mathrm{~V} 9+\mathrm{R} 4$ & $22 /$ enero, $11 /$ febrero \\
11 & & $\mathrm{~V} 9+\mathrm{R} 4+\mathrm{R} 5.3$ & $22 /$ enero, $11 /$ febrero, 3/marzo \\
12 & Epoxiconazol + & $\mathrm{V} 9+\mathrm{R} 5.3$ & $22 /$ enero, 3/marzo \\
13 & piraclostrobina & $\mathrm{R} 4+\mathrm{R} 5.3$ & $11 /$ febrero, 3/marzo \\
14 & & $\mathrm{R} 4$ & $11 /$ febrero \\
15 & & $\mathrm{R} 5.3$ & $3 /$ marzo \\
\hline 16 & & $\mathrm{~V} 9+\mathrm{R} 3+\mathrm{R} 5.1+\mathrm{R} 6$ & $22 /$ enero, 6/febrero, 21/febrero, 3/marzo \\
\hline
\end{tabular}

*De acuerdo con Fehr \& Caviness (1977) 
cultivada en el campo experimental de la Facultad de Agronomía y Medicina Veterinaria - FAMV de la Universidad de Passo Fundo - UPF, RS ubicada entre las coordenadas geográficas $28^{\circ} 07^{\prime}$ y $28^{\circ} 25^{\prime}$ de latitud Sur y $52^{\circ} 17^{\prime}$ y $52^{\circ} 41^{\prime}$ de longitud oeste. El cultivo fue establecido el 05/12/2009, a través de siembra directa sobre restos culturales de trigo (Triticum aestivum L.). Se utilizó un espaciamiento de $0.45 \mathrm{~m}$ entre líneas y una densidad de 14 plantas por metro lineal. Las semillas fueron tratadas con una mezcla comercial (Standak Top ${ }^{\circledR}, 100 \mathrm{~mL} 100 \mathrm{~kg}^{-1}$ de semilla) de insecticida fipronil y dos fungicidas piraclostrobina y tiofanato metílico. La fertilización fue realizada con una aplicación de $300 \mathrm{~kg}$ $\mathrm{ha}^{-1}$ de la fórmula 5-25-20 (N-P2O5-K2O). Los demás tratos culturales fueron realizados en función de las indicaciones técnicas para el cultivo de soya.

El área experimental contenía 64 parcelas, cada una con un área de $15.75 \mathrm{~m}^{2}$ y un promedio de 490 plantas establecidas. El delineamiento experimental fue en bloques casualizados con 16 tratamientos y cuatro repeticiones cada una. Tales parcelas fueron sometidas a una, dos o tres aplicaciones de los fungicidas tebuconazol (Folicur ${ }^{\circledR}$ CE, 0.50 L.ha ${ }^{-1}$ ) y epoxiconazol + piraclostrobina $\left(\right.$ Opera $\left.{ }^{\circledR}, 0.50 \mathrm{~L} \cdot \mathrm{ha}^{-1}\right)$ en la parte aérea, aparte de un testigo no tratado y de un tratamiento con cuatro aplicaciones de la mezcla señalada anteriormente (Cuadro 1). Las mencionadas aplicaciones fueron realizadas con la finalidad de generar gradientes de área foliar en el área experimental, las cuales fueron iniciadas en el estadio fenológico V9 (rama principal con nueve trifolios expandidos) (Fehr y Caviness, 1977).
En el estadio fenológico R7.1 (1 a 50\% de amarillamiento de hojas y vainas) (Fehr y Caviness, 1977), dos plantas fueron colectadas al azar en cada una de las 64 parcelas y sus folíolos removidos e inmediatamente acondicionados en sacos plásticos, mantenidos a la sombra. De inmediato para evitar la deshidratación, los folíolos fueron pesados en una balanza digital (modelo Sartorius) para la determinación del peso fresco. Posteriormente, los folíolos fueron medidos su área a través de un integralizador digital de área foliar (Licor modelo LI-3000A). Subsiguientemente, los mismos fueron acondicionados en fundas de papel (capacidad de $5.0 \mathrm{~kg}$ ) y deshidratados en una estufa de circulación forzada de aire, a $65^{\circ} \mathrm{C}$ por 48 horas, para la determinación del peso seco, en gramos.

Estas evaluaciones fueron realizadas por estratos en las plantas de soya (inferior, medio y superior), siendo divididos en tres partes iguales de acuerdo al tamaño de cada una las plantas de soya. Para mejor entendimiento del lector, se explica en la Figura 1.

Los datos fueron expresados como área foliar total $\left(\mathrm{cm}^{2}\right)$ y peso total $(\mathrm{g})$ por planta. El análisis estadístico realizado fue una regresión lineal, utilizando el área media por el integralizador como variable dependiente (y) y los pesos fresco y seco como variables independientes $(\mathrm{x})$.

Los análisis de regresión fueron realizados en el programa SAS 9.0 (SAS, 2002) y las figuras en Microsoft Excel.
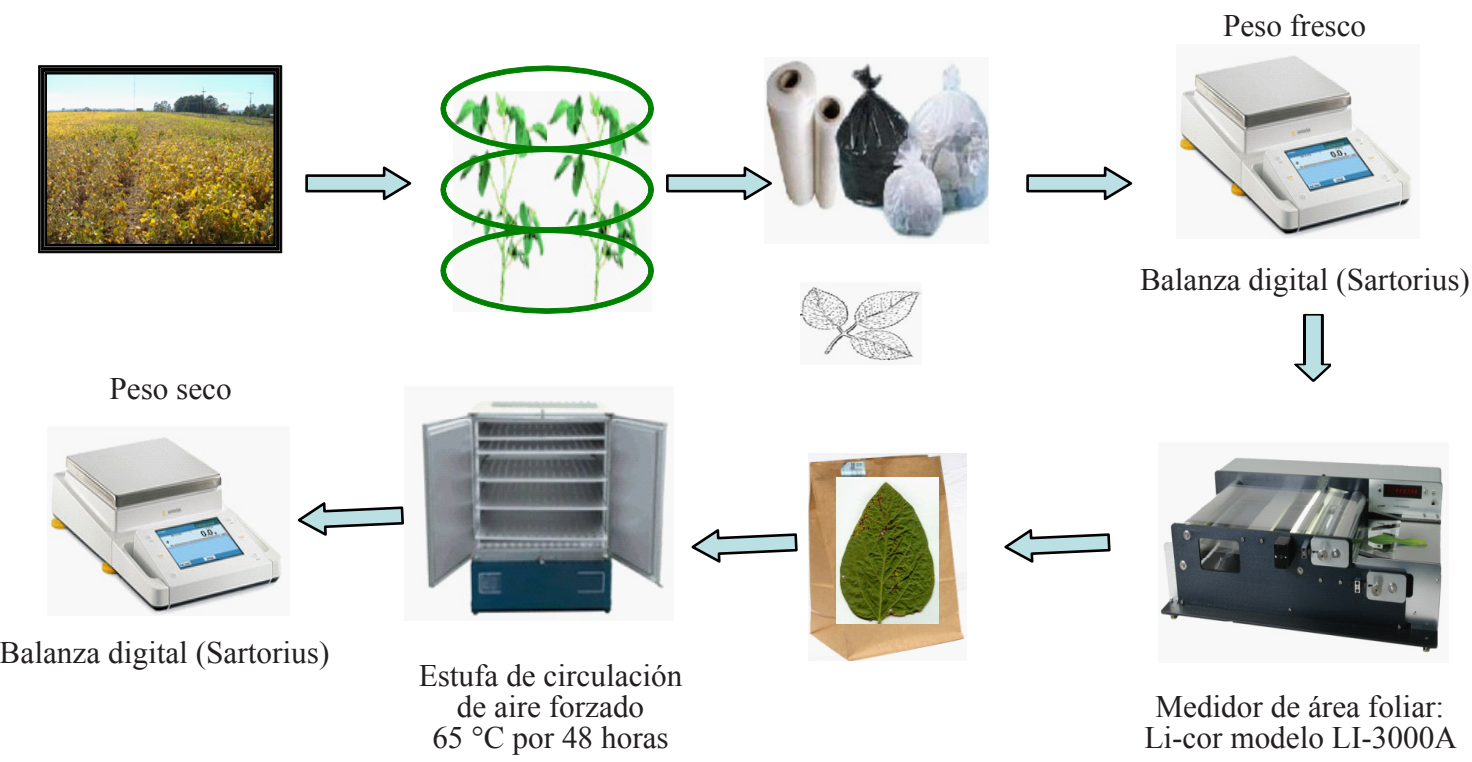

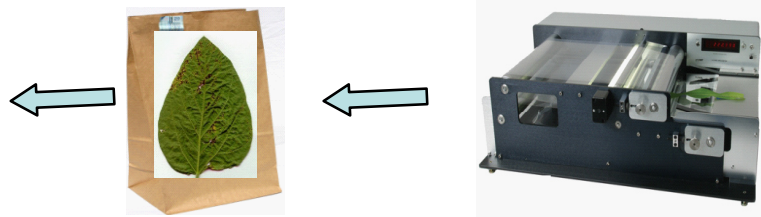

Medidor de área foliar: Li-cor modelo LI-3000A

Figura 1. Proceso de la medición del área foliar y el peso de masa fresca y seca de hojas en cada uno de los estratos de plantas de soya, cultivar Nidera 5909 RG. Passo Fundo/RS, época de verano 2009/10. 


\section{Resultados y Discusión}

L as relaciones entre peso de materia seca y área foliar (Figura 2) fueron significativas ( $<<0.0001$ e $\mathrm{R}^{2} \mathrm{de}$ 0.74 a 0.97 ) para el cultivar utilizado.

Con base en las ecuaciones generadas, cada gramo en peso fresco representa una variación de 33.01 a $46.50 \mathrm{~cm}^{2}$ en el área foliar. Esta variación fue menor en el estrato inferior (33.01) y mayor en el medio (45.21) y superior (46.50), siendo 45.53 la media de toda la planta. En relación al peso de materia seca (Figura 3), las ecuaciones también fueron altamente significativas, y las variaciones en el área foliar para cada unidad de

a)

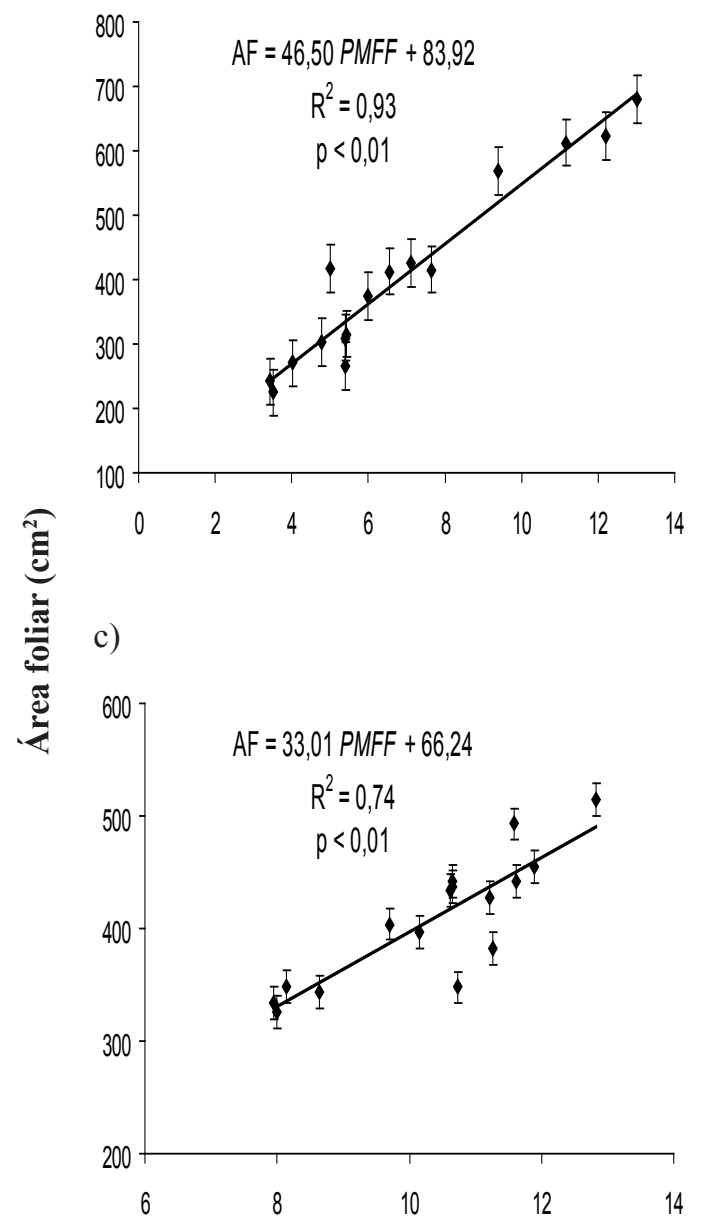

peso subieron para 104.65 (estrato inferior), 168.50 (estrato medio), 196.53 (estrato superior) y 176.17 $\mathrm{cm}^{2}$ en el área foliar (total de toda la planta).La buena relación $\left(\mathrm{p}<0.0001\right.$ e $\mathrm{R}^{2}$ de 0.74 a 0.97$)$ del peso de materia fresca y seca con el área foliar permite que esta herramienta pueda ser usada en trabajos donde se tenga que evaluar el área foliar en el cultivo de soya. Con todo, hay pocas referencias con respecto al uso de la metodología propuesta en otros cultivos. Uno de ellos fue desarrollado por Barrantes y Gracia (1989), los cuales observaron buena correlación entre peso de materia seca de hojas y área foliar en Pinus sylvestris $\mathrm{L}$.

b)

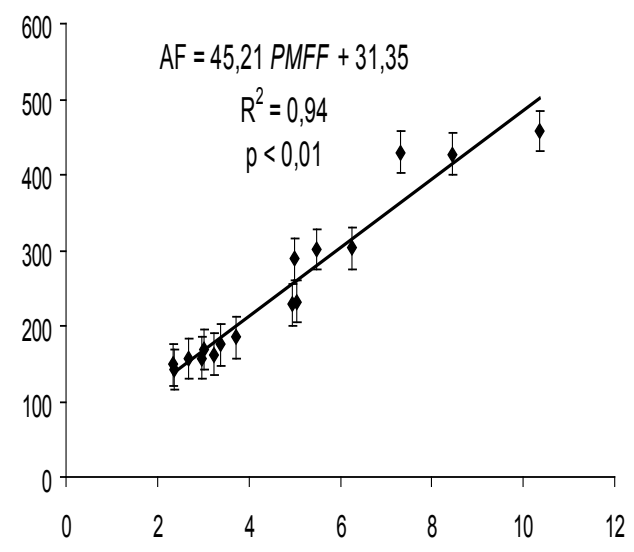

d)

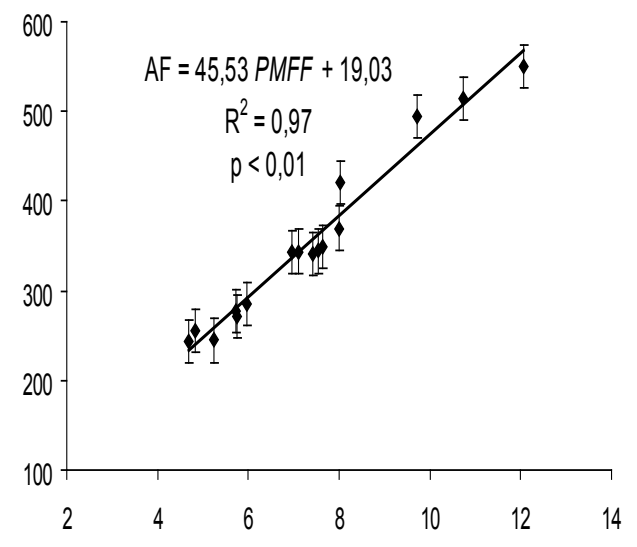

Peso de materia fresca de hojas (g)

Figura 2. Relación entre el peso de materia fresca de hojas (PMFF) y el área foliar (AF) en el estrato inferior (a), medio (b) y superior (c) de plantas de soya, cultivar Nidera 5909 AG. La letra "d" representa la media de la planta. Passo Fundo/RS, Zafra 2009/10 
a)
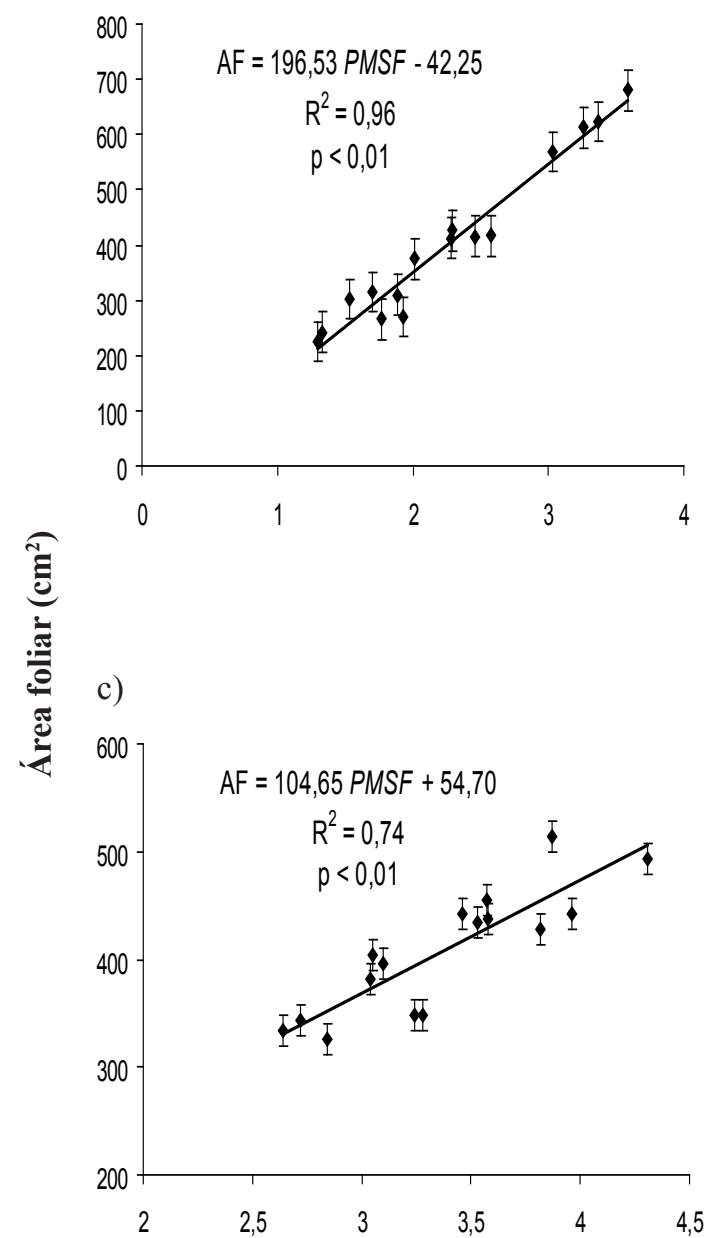

b)

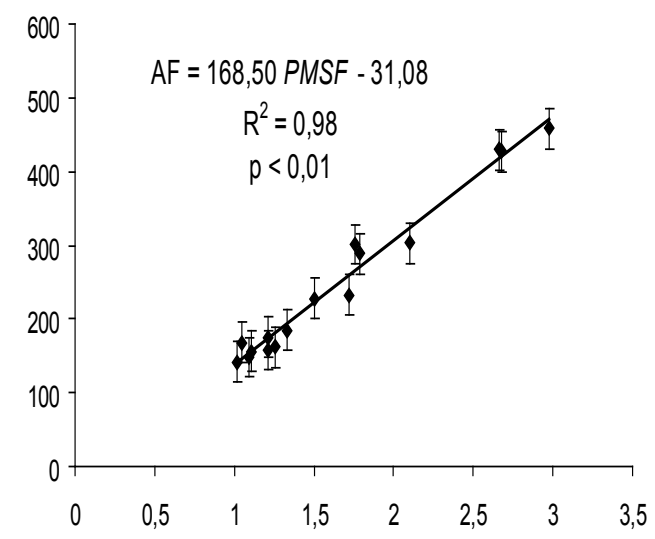

d)

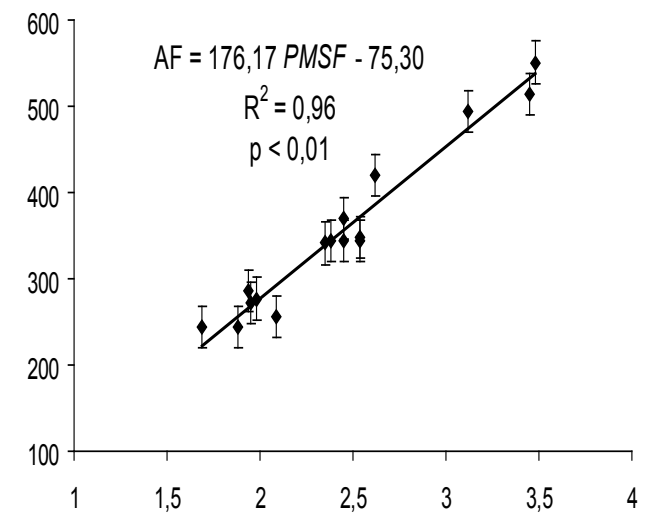

Peso de materia seca de hojas (g)

Figura 3. Relación entre el peso de materia seca de hojas (PMSF) y el área foliar (AF) en el estrato inferior (a), medio (b) y superior (c) de plantas de soya, cultivar Nidera 5909 AG. La letra "d" representa la media de la planta. Passo Fundo/RS, Zafra 2009/10

\section{Conclusiones}

$\mathrm{L}^{2}$ a relación de los pesos fresco o seco con el área foliar es significativa, pudiendo ser utilizada para estimar el área foliar de la planta y consecuentemente, el índice de área foliar de un cultivo de soya. Así también, se demuestra que el uso de peso seco parece más viable, pues no requiere pesar las plantas inmediatamente después de la cosecha.

La utilización futura de esta herramienta requiere estudios adicionales con otros cultivares, con la finalidad de verificar si el comportamiento es similar.

\section{Agradecimientos}

A la Secretaría Nacional de Educación Superior, ACiencia, Tecnología e Innovación del Ecuador - SENESCYT por la beca concedida al primer autor, para estudios de Maestría en Agronomía con Área en Fitopatología en la Universidad de Passo Fundo, RS, Brasil. 


\section{LITERATURA CITADA}

Aquino, V. M., F. M. Shokes, R. D. Berger, D. W. Gorbet and T. A. Kucharak. 1992. Relationship among late leafspot, healthy leaf area duration, canopy reflectance, and pod yield of peanut. Phytopathology. 82: 546-552.

Barrantes, O. y C. A. Gracia. 1989. Estimación del área foliar a partir de la superficie de albura en Pinus sylvestris. Options Méditerranéennes-Serie Séminaires. 3: 53-56.

Barros, H. B., M. M. Santos, J. M. Pelúzio, R. N. C. Rocha, e R. R. Silva. 2002. Desfolha na produção de soja (Glycine max M-SOIL 109), cultivada no cerrado, em Gurupi-TO, Brasil. Bioscience Journal. 18 (2): 5-10.

Bergamin Filho, A. e L. Amorim. 1996. Doenças de Plantas Tropicais: Epidemiologia e Controle Econômico. São Paulo, Ceres.

Díaz, C. G., L. D. Ploper, M. R. Galvez, y B. Gonzalez. 2005. Efecto de las enfermedades de fin de ciclo en el crecimiento de distintos genotipos de soja relacionado a la fecha de siembra. Agriscientia 21(1): 1-7.

Fehr, W. and S. E. Caviness. 1977. Stages of soybean development Agriculture and Home Economics Exp. Sta. Iowa University of Sci. and Tech. Special Report 80.

Heiffig, L. S., G. M. S. Câmara, L. A. Marques, D. B. Pedroso, e S. M. S. Piedade. 2006. Fechamento e índice de área foliar da cultura da soja em diferentes arranjos espaciais. Bragantia. 65: 285-295.
Jesus Junior, W. C., F. X .R. Vale, R. R. Coelho, and L. Costa. 2001. Comparison of two methods for estimating leaf area index on common bean. Agronomy Journal. 93: 989-991.

Pelluzio, J. M., H. B. Barros, R. N. C. Rocha, R. R. Silva, e I. R. Nascimento. 2002. Influência do desfolhamento artificial no rendimento de grãos e componentes de produção da soja [Glycine max (L.) Merrill]. Ciênc. Agrotec. 26 (6): 1197-1203.

Rezende de Oliveira, M. L. 2007. Estimativa da desfolha e o índice de área foliar no patossistema sojaferrugem asiática utilizando o programa QUANT. Tesis presentada para la obtención de Magister Scientiae. Universidad de Viçosa, Brasil.

SAS/STAT® Versão 9.0 del sistema SAS para Windows, copyright 2002 SAS Institute Inc., Cary, NC, USA.

Seem, R. C. 1988. The meauserement and analysis of the effects of crop development on epidemics. In: Kranz, J. and Rotem, J. (Eds.) Experimental techniques in plant disease epidemiology. Springer-Verlag, Heidelberg.

Van Der Plank, J. E. 1963. Plant Diseases: Epidemics and Control. Academic Press, New York \& London.

Waggoner, P. E. and R. D. Berger. 1987. Defoliation, disease, and growth. Phytopathology 77: 393398. 